\title{
Calculated oscillator strengths for the strongest lines of cosmochronological interest in the visible spectrum of singly ionized uranium (U II)
}

\author{
S. Gamrath, ${ }^{1}$ P. Palmeri ${ }^{1}$ and P. Quinet ${ }^{1,2 \star}$ \\ ${ }^{1}$ Physique Atomique et Astrophysique, Université de Mons, B-7000 Mons, Belgium \\ ${ }^{2}$ IPNAS, Université de Liège, Sart Tilman, B-4000 Liège, Belgium
}

Accepted 2018 August 8. Received 2018 August 03; in original form 2018 May 4

\begin{abstract}
This work reports new calculations of radiative parameters for spectral lines of cosmochronological interest in singly ionized uranium. More precisely, a pseudo-relativistic HartreeFock model including core-polarization effects has been employed to compute the oscillator strengths corresponding to the strongest $U_{\text {II }}$ transitions in the visible wavelength range. The results obtained in the present investigation appear to be in reasonable agreement with the most accurate experimental data, reducing in that way the large scattering observed between the formerly published $g f$ values. This allowed us to provide a list of 38 lines that could be used by astrophysicists as cosmochronometers in future studies to determine stellar ages from uranium radioactive decay.
\end{abstract}

Key words: atomic data-atomic processes.

\section{INTRODUCTION}

Oscillator strengths for radiative transitions in singly ionized uranium are of paramount importance in astrophysics, in particular in cosmochronology. It is indeed well known that the use of a longlived radioisotope can be used to estimate the age of a star. Up until a few years ago, ${ }^{232} \mathrm{Th}$, with a half-life of $14 \mathrm{Gyr}$, was used to date galactic stars (Butcher 1987, François et al. 1993, Cowan et al. 1999) but this radioisotope decays by approximately twice the lifetime of the Universe, and, as pointed out by Goriely \& Clerbaux (1999), new accurate observations of heavy radioactive elements would be necessary to improve the accuracy of cosmochronometry analyses. More particularly, ${ }^{238} \mathrm{U}$, with a half-life of $4.5 \mathrm{Gyr}$, represents a much more precise age indicator. Furthermore, the ratio U/Th might be a better cosmochronometer than either previously used $\mathrm{Th} / \mathrm{Eu}$ or Th/Dy ratios, because of the much smaller mass difference between thorium and uranium than between either one of these two elements and the lighter lanthanides.

However, uranium is very hard to detect in stars. In 2001, Cayrel et al. (2001a) reported the first observation of a spectral line at a wavelength of $3859.57 \AA$, from singly ionized uranium, in the very metal-poor star BPS CS31082-001. This star, also called Cayrel's star, was found to be more metal deficient than the globular clusters, and was likely born in the Galaxy during very early times. This measurement, carried out with the high-resolution UVES spectrograph of the ESO/VLT telescope, gave rise to the determination of

^E-mail: Pascal.QUINET@umons.ac.be the age of formation of $\mathrm{U}$ and Th in the early Galaxy, using, for the first time, the U/Th ratio. The derived uranium abundance yielded an age $12.5 \pm 3 \mathrm{Gyr}$, which led to the best estimate of the age of the Galaxy and consequently provided a lower limit to the age of the Universe. However, as mentioned by Cayrel et al. (2001b), the accuracy of this uranium dating is still affected by uncertainties in the measured abundance ratio, and in the calculated U/Th production ratio. The improvement of this situation depends not only on a better estimation of the production ratio U/Th, on advances in nuclear astrophysics models and on the investigation of other stars in which $\mathrm{U}$ and $\mathrm{Th}$ can be detected, but also on a comprehensive knowledge of the radiative properties for the potentially observable spectral lines, notably for the strongest U II electric dipole transitions.

To our knowledge, there have been very few available calculations of radiative parameters for singly ionized uranium so far. The main reason is that the complexity of the strongly mixed states involved in the lowest configurations, with five electrons distributed on the $5 \mathrm{f}, 6 \mathrm{~d}, 7 \mathrm{~s}$, and $7 \mathrm{p}$ subshells, and the fragmentary knowledge of the experimental spectrum of this ion made theoretical computations extremely difficult. Most of the available oscillator strengths were deduced from quite old line intensities measured on arc spectra (Meggers, Corliss \& Schribner 1961, Corliss \& Bozman 1962, Voigt 1975, Corliss 1976, Kurucz 1995) if we except the results published by Nilsson et al. (2002) who obtained accurate $g f$ values by combining branching fraction measurements with laboratory lifetimes determined using laser spectroscopy (Lundberg et al. 2001). These latter data concern however only a small number of strong $U$ II lines. 
This lack of reliable radiative parameters in singly ionized uranium justifies the work reported in this paper. An additional motivation was also found in the new analysis of the experimental spectrum recently published for this ion by Meftah et al. (2017). More precisely, we carried out detailed calculations of oscillator strengths for the strongest U II transitions using the pseudo-relativistic HartreeFock (HFR) method (Cowan 1981) with core-polarization corrections (HFR+CPOL; Quinet et al. 1999, 2002, Quinet 2017). This allowed us to draw up a list of 38 visible spectral lines that could be used in cosmochronology.

\section{AVAILABLE ATOMIC DATA IN U II}

In their critical compilation related to actinides, Blaise \& Wyart (1992) listed some preliminary U II energy levels, using the emission lines listed by Steinhaus et al. (1971) and Palmer, Keller \& Engleman (1980), thus updating the previous estimates published by Brewer (1971). An uranium hollow-cathode Fourier transform spectrum between 1800 and $42000 \mathrm{~cm}^{-1}$ was then combined with previous visible and ultraviolet spectra by Blaise et al. (1994) who were able to determine the numerical values for 354 and 809 energy levels belonging to the four odd configurations $5 \mathrm{f}^{3} 7 \mathrm{~s}^{2}, 5 \mathrm{f}^{3} 6 \mathrm{~d} 7 \mathrm{~s}, 5 \mathrm{f}^{3} 6 \mathrm{~d}^{2}$, $5 \mathrm{f}^{4} 7 \mathrm{p}$, and to the six even configurations $5 \mathrm{f}^{4} 7 \mathrm{~s}, 5 \mathrm{f}^{4} 6 \mathrm{~d}, 5 \mathrm{f}^{2} 6 \mathrm{~d}^{2} 7 \mathrm{~s}$, $5 f^{2} 6 d 7 s^{2}, 5 f^{3} 7 s 7 p, 5 f^{3} 6 d 7 p$, respectively.

More recently, Meftah et al. (2017) used a parametric approach based on the Cowan (1981) codes to classify the lowest energy levels in singly ionized uranium. This work led to the identification of 253 levels belonging to the odd-parity configurations $5 f^{3} 7 s^{2}+5 f^{3} 6 d 7 s+$ $5 f^{3} 6 d^{2}+5 f^{4} 7 p+5 f^{5}$, using 24 adjustable and 64 constrained radial parameters, with a root mean square $(\mathrm{rms})$ deviation of $60 \mathrm{~cm}^{-1}$. In the even parity, 125 levels were classified using a multiconfiguration basis including $5 f^{4} 7 s+5 f^{4} 6 d+5 f^{2} 6 d^{2} 7 s+5 f^{2} 6 d 7 s^{2}+5 f^{2} 6 d^{3}$ by 22 free parameters with an rms deviation of $84 \mathrm{~cm}^{-1}$. Moreover, a separate semi-empirical model, including only the higher even configurations $5 \mathrm{f}^{3} 7 \mathrm{~s} 7 \mathrm{p}$ and $5 \mathrm{f}^{3} 6 \mathrm{~d} 7 \mathrm{p}$, led to the tentative classification of 12 energy levels within these configurations. Unfortunately, for these two configurations, the semi-empirical parametric fitting has to be limited to the adjustment of average energies and spin-orbit $\zeta_{5 f}$ integrals, the quantitative evaluation of configuration interaction effects within the whole group $5 f^{4}(7 s+6 d)+5 f^{2}(6 d+7 s)^{3}+5 f^{3} 7 s 7 p$ $+5 f^{3} 6 d 7 p$ having been tried unsuccesfully. Finally, the parametric study of Meftah et al. (2017) allowed them to re-investigate the high resolution ultraviolet spectrum of uranium recorded about $30 \mathrm{yr}$ earlier at the Meudon Observatory. This led to the classification of 451 additional $U$ II lines in the wavelength region 2344-2955 $\AA$ and the identification of one new level belonging to the $5 f^{3} 6 \mathrm{~d} 7 \mathrm{p}$ configuration.

As regards the radiative decay rates, the first measurements of relative line intensities in U II were obtained from emission arc spectra by Meggers et al. (1961), Corliss and Bozman (1962), Voigt (1975), and Corliss (1976). In the atlas of uranium lines published by Palmer et al. (1980), relative intensities were listed for $4928 \mathrm{U}_{\mathrm{I}}$ and $431 \mathrm{U}$ II emission spectral lines between 11000 and $26000 \mathrm{~cm}^{-1}$. The oscillator strengths of the lines at $\lambda=3859.571 \AA$ and $\lambda=$ 4050.041 A were later determined by Chen \& Borzileri (1981) who combined experimental lifetime measurements of the upper levels with unpublished branching fractions. Oscillator strengths were also reported for about 100 U II lines by Henrion, Fabry \& Remy (1987) from relative intensities measured on a hollow-cathode lamp spectrum. In his data base, Kurucz (1995) listed oscillator strengths and transition probabilities for many U II lines based on the experimental data reported by Meggers et al. (1961), Corliss (1976), and Chen and Borzileri (1981). Finally, about 20 yr ago, accurate radiative lifetimes were measured by Lundberg et al. (2001), using laser-induced fluorescence technique, for six even-parity energy levels of singly ionized uranium located at $23315.090 \mathrm{~cm}^{-1}$ $(J=9 / 2), 24684.132 \mathrm{~cm}^{-1}(J=9 / 2), 25714.049 \mathrm{~cm}^{-1}(J=13 / 2)$, $26191.309 \mathrm{~cm}^{-1}(J=13 / 2), 28154.450 \mathrm{~cm}^{-1}(J=11 / 2)$, and $30341.675 \mathrm{~cm}^{-1}(J=15 / 2)$. Experimental oscillator strengths for $57 \mathrm{U}_{\text {II }}$ lines in the region $3500-6700 \AA$ were then determined by combining these latter radiative lifetimes with new branching fractions deduced from line intensity measurements performed using Fourier transform spectroscopy (Nilsson et al. 2002).

\section{OSCILLATOR STRENGTH CALCULATIONS}

Reliable atomic structure calculations in U II must take intravalence, core-valence, and core-core correlations into account, in addition to relativistic effects. In our work, we used the pseudo-relativistic HFR method (Cowan, 1981), in which the largest part of the intravalence correlation is represented by explicitly including a set of electronic configurations in the physical model, while the effects of core-excited configurations are modelled by a CPOL potential. As described e.g. by Quinet et al. (1999, 2002) and Quinet (2017) for atomic systems with $n$ valence electrons, the one-particle operator of this potential can be written as

$V_{P 1}=-\frac{1}{2} \alpha_{\mathrm{d}} \sum_{i=1}^{n} \frac{r_{i}^{2}}{\left(r_{i}^{2}+r_{c}^{2}\right)^{3}}$,

where $\alpha_{\mathrm{d}}$ is the dipole polarizability of the ionic core and $r_{\mathrm{c}}$ is a suitable cut-off radius which is arbitrarily chosen as a measure of the size of the ionic core.

In addition, the interaction between the modified electric fields experienced by the valence electrons gives rise to a two-particle contribution given by

$V_{P 2}=-\alpha_{\mathrm{d}} \sum_{i>j} \frac{\vec{r}_{i} \cdot \vec{r}_{j}}{\left[\left(r_{i}^{2}+r_{\mathrm{c}}^{2}\right)\left(r_{j}^{2}+r_{\mathrm{c}}^{2}\right)\right]^{3 / 2}}$.

For consistency, in this HFR+CPOL method, the transition radial integral $\left\langle P_{n l}|r| P_{n^{\prime} l^{\prime}}\right\rangle$ has also to be replaced by

$\left\langle P_{n l}\left|r\left(1-\frac{\alpha_{\mathrm{d}}}{\left(r^{2}+r_{\mathrm{c}}^{2}\right)^{3 / 2}}\right)\right| P_{n^{\prime} l^{\prime}}\right\rangle$.

Moreover, in order to allow for a more accurate treatment of the penetration of the core by the valence electrons, a further correction has been included in our model. This correction, originally introduced by Hameed, Herzenberg \& James (1968) and Hameed (1972), consists in the addition of the core-penetration term

$\frac{1}{r_{\mathrm{c}}^{3}} \int_{0}^{r_{\mathrm{c}}} P_{n l}(r) r P_{n^{\prime} l^{\prime}}(r) \mathrm{d} r$.

to the integral

$$
\left\langle P_{n l}(r)\left|\frac{r}{\left(r^{2}+r_{c}^{2}\right)^{3 / 2}}\right| P_{n^{\prime} l^{\prime}}(r)\right\rangle
$$

appearing in equations (2) and (3).

In this study, the configurations retained in the calculations were those presented by Meftah et al. (2017) as being the most interacting ones in the lowest part of the energy level spectrum, namely $5 \mathrm{f}^{3} 7 \mathrm{~s}^{2}$, $5 \mathrm{f}^{3} 6 \mathrm{~d} 7 \mathrm{~s}, 5 \mathrm{f}^{3} 6 \mathrm{~d}^{2}, 5 \mathrm{f}^{4} 7 \mathrm{p}, 5 \mathrm{f}^{5}$ for the odd parity, and $5 \mathrm{f}^{4} 7 \mathrm{~s}, 5 \mathrm{f}^{4} 6 \mathrm{~d}$, $5 f^{2} 6 d^{2} 7 s, 5 f^{2} 6 d 7 s^{2}, 5 f^{2} 6 d^{3}, 5 f^{3} 7 s 7 p, 5 f^{3} 6 d 7 p$ for the even parity. This represents a total of 3237 and 6039 energy levels in each parity, respectively. 


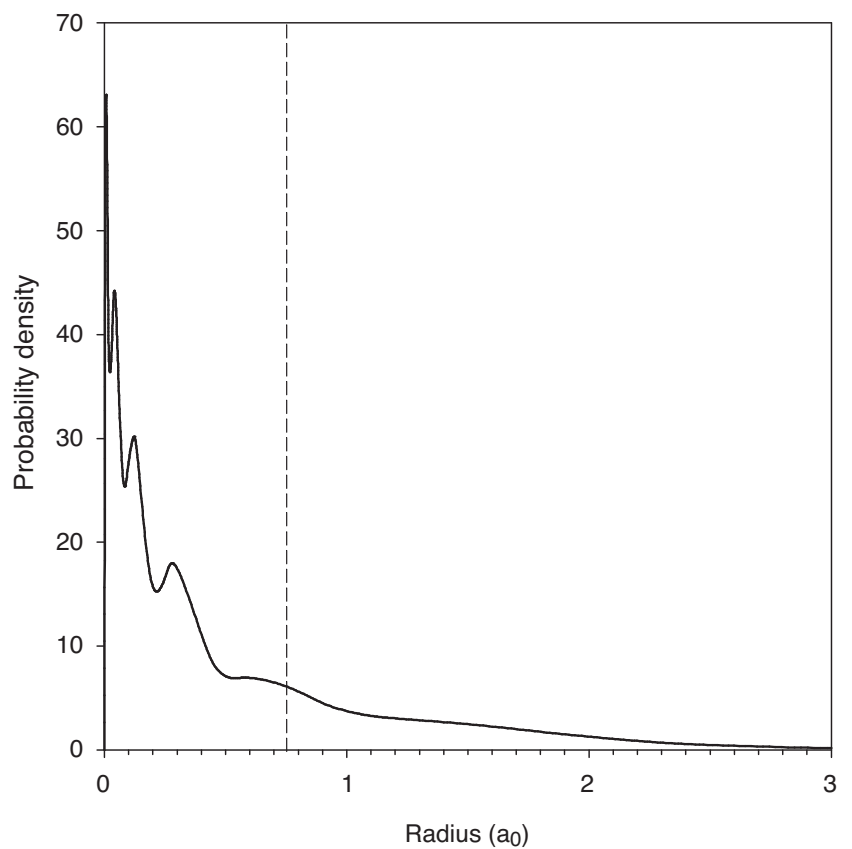

Figure 1. Electron probability density $\left(\sum P_{n l}^{2}\right)$ of the ionic core in the ground configuration $\left(5 \mathrm{f}^{3} 7 \mathrm{~s}^{2}\right)$ of $\mathrm{U}$ II. The value of the cut-off radius used in the HFR+CPOL calculations $\left(r_{\mathrm{c}}=0.75 \mathrm{a}_{0}\right)$ is also shown in the figure. It represents the distance at which the electron probability density falls to 10 per cent of its maximum value, as suggested by Hameed (1972).

Since the configurations mentioned hereabove explicitly include a part of the correlation out of the $5 \mathrm{f}^{2}$ ionic core, CPOL effects were considered using the dipole polarizability, $\alpha_{\mathrm{d}}$, equal to $9.79 \mathrm{a}_{0}^{3}$ as tabulated by Fraga, Karwowski \& Saxena (1976) for the U V ion. However, as mentioned, for example, by Hibbert (1982), the cut-off radius, $r_{\mathrm{c}}$ is not unambiguously defined. In this work, we used $r_{\mathrm{c}}=$ $0.75 \mathrm{a}_{0}$ which represents the distance at which the total probability density of the ionic core orbitals falls to 10 per cent of its maximum value, as suggested by Hameed (1972). Fig. 1 shows the calculated probability density of the core in the ground configuration $5 \mathrm{f}^{3} 7 \mathrm{~s}^{2}$, together with the $r_{\mathrm{c}}$ value used in the computations. We noticed that, when using these $\alpha_{\mathrm{d}}$ and $r_{\mathrm{c}}$ CPOL parameters, all the $\langle 6 \mathrm{~d}|r| 7 \mathrm{p}\rangle$ and $\langle 7 \mathrm{~s}|r| 7 \mathrm{p}\rangle$ electric dipole transition radial integrals were reduced by $20-25$ per cent. However, for the singly ionized uranium, the analytical CPOL correction to the dipole operator introduced in equation (3) is no longer valid for transitions involving $5 f$ electrons, the latter being deeply imbedded inside the closed $6 \mathrm{~s}^{2} 6 \mathrm{p}^{6}$ subshells, as shown in Fig. 2. Instead, in order to take polarization effects into account for the $5 f-6 \mathrm{~d}$ transitions, the uncorrected $\langle 5 \mathrm{f}|r| 6 \mathrm{~d}\rangle$ radial integrals were scaled down by the factor 0.80 , in a similar way to that used for computing the $4 \mathrm{f}-5 \mathrm{~d}$ transitions in lowly ionized lanthanides (see e.g. Biémont et al. 2001; Li et al. 2001; Zhang et al. 2001; Biémont, Quinet \& Ryabchikova 2002).

Finally, in order to reduce as much as possible the differences between calculated and available experimental energy levels, the fitted radial parameters reported by Meftah et al. (2017) were adopted for the five odd- and the seven even-parity configurations included in our physical model. As a reminder, for the odd configurations, Meftah et al. were able to fit up to 253 experimental energy levels with a final rms deviation of $60 \mathrm{~cm}^{-1}$ using 22 free and 64 constrained parameters among the average energies $\left(E_{\mathrm{av}}\right)$, the monoconfiguration and configuration interaction (CI) electrostatic integrals $\left(F^{k}\right.$,

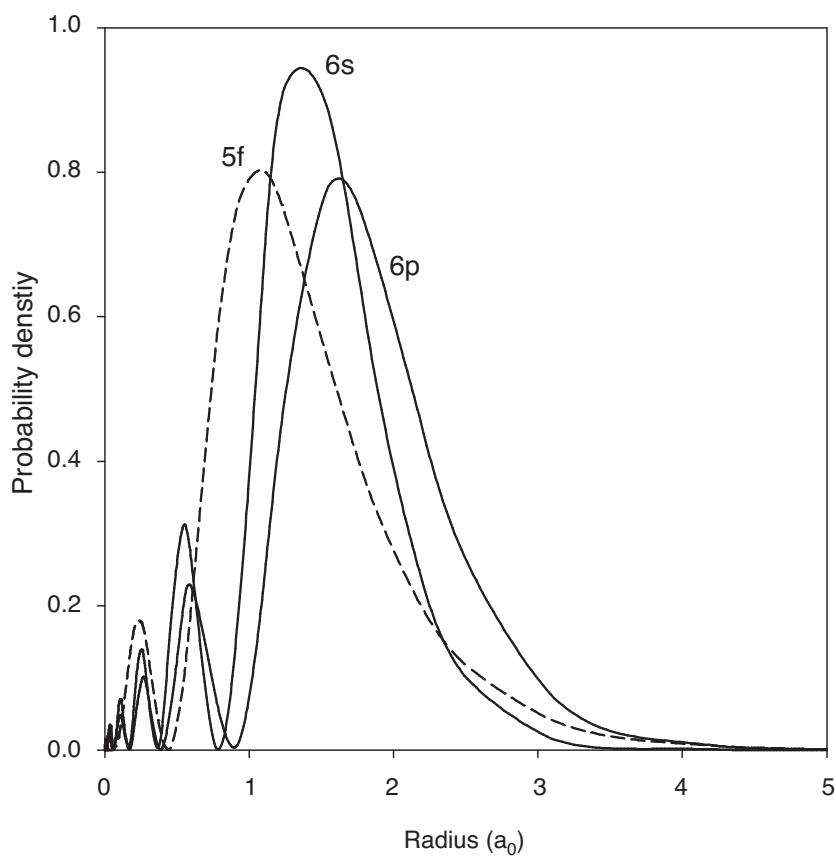

Figure 2. Electron probability densities $\left(P_{n l}^{2}\right)$ of the outermost ionic core orbitals in U II, showing the collapse of $5 \mathrm{f}$ into the closed $6 \mathrm{~s}^{2} 6 \mathrm{p}^{6}$ subshells.

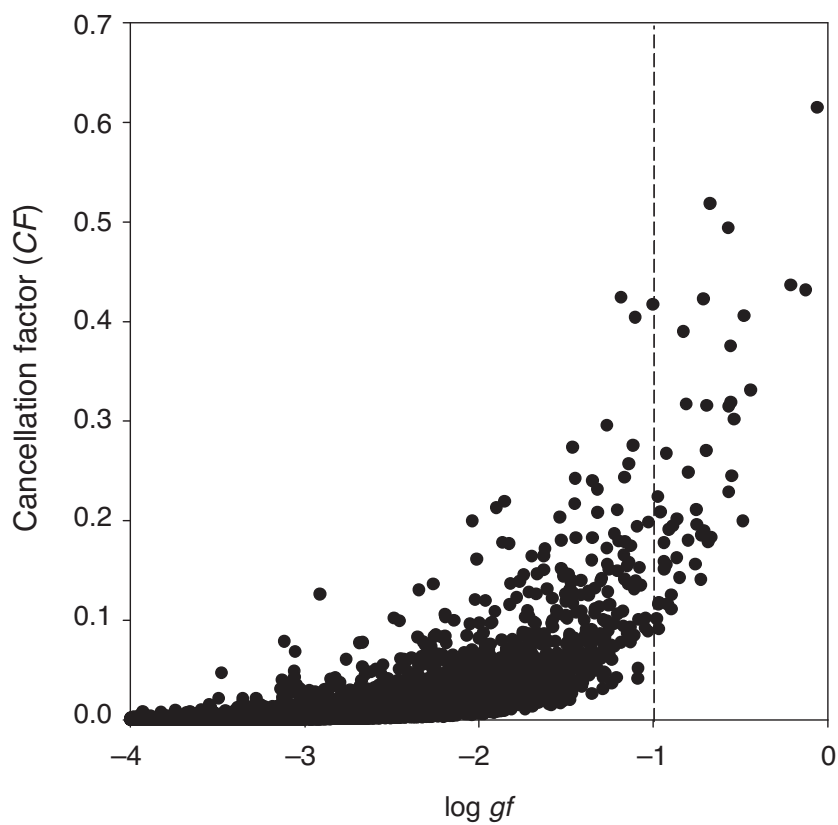

Figure 3. Cancellation factors plotted as a function of $\log g f$ values as obtained in this work for $\mathrm{U}$ II spectral lines. CF values smaller than typically 0.05 indicate that the corresponding $f$ values may be affected by large percentage errors. Strong U II transitions with $\log g f>-1$ do not appear to be affected by cancellation effects.

$\left.G^{k}, R^{k}\right)$, the spin-orbit parameters $\left(\zeta_{n l}\right)$, the effective interaction operators $(\alpha, \beta, \gamma)$ and Slater forbidden parameters within the whole group of configurations $5 f^{3} 7 s^{2}+5 f^{3} 6 d 7 s+5 f^{3} 6 d^{2}+5 f^{4} 7 p+5 f^{5}$. The semi-empirical fitting procedure was a little less satisfactory in the even parity, Meftah et al. (2017) being forced to separate their parametric analysis into two groups of configurations, i.e. $5 \mathrm{f}^{4} 7 \mathrm{~s}+$ 
Table 1. Strongest visible spectral lines in U II. The transitions listed are limited to those for which the $\log g f$ values, computed in this work, are greater than -1.0 .

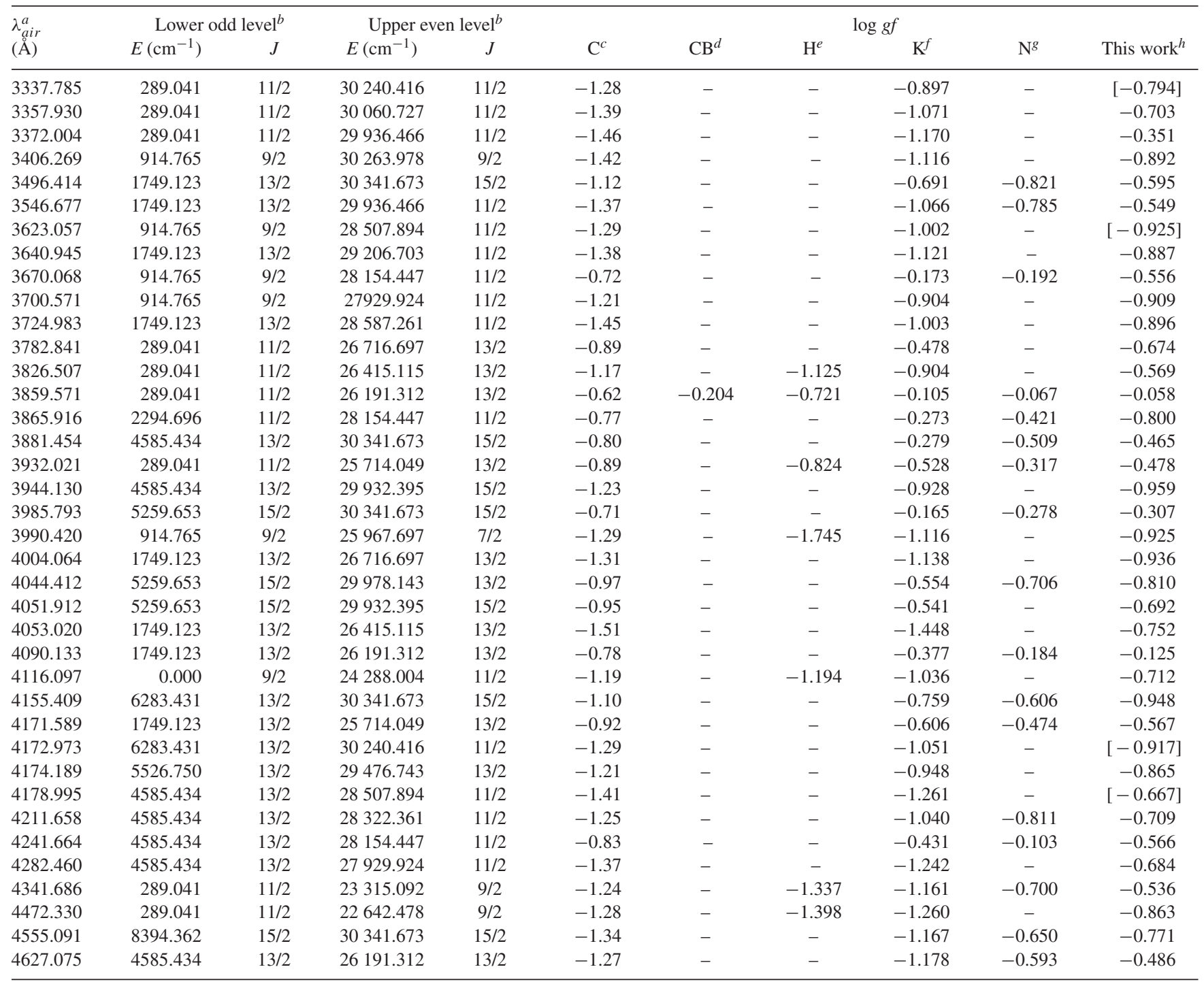

Notes. ${ }^{a}$ Experimental wavelengths from Kurucz (1995).

${ }^{b}$ Experimental energy levels from Blaise et al. (1994) and Meftah et al. (2017).

${ }^{c}$ Values from Corliss (1976).

${ }^{d}$ Values from Chen and Borzileri (1981).

${ }^{e}$ Values from Henrion et al. (1987).

${ }^{\mathrm{f}}$ Values from Kurucz (1995).

${ }^{g}$ Values from Nilsson et al. (2002).

${ }^{h}$ Values between square brackets correspond to uncertain line identifications in the calculations (see text).

$5 f^{4} 6 d+5 f^{2} 6 d^{2} 7 s+5 f^{2} 6 d 7 s^{2}+5 f^{2} 6 d^{3}$, on the one hand, and $5 f^{3} 7 s 7 p$ $+5 \mathrm{f}^{3} 6 \mathrm{~d} 7 \mathrm{p}$, on the other hand. For the first group, an rms deviation of $84 \mathrm{~cm}^{-1}$ was found for 125 energy levels fitted with 22 radial parameters of the same type as those used in the odd parity. However, the interpretation of energy level structure of the second group with the same parametric method could only be carried out by adjusting only the average energies $E_{\mathrm{av}}$ and spin-orbit $\zeta_{5 f}$ integrals, leading to an rms deviation of $441 \mathrm{~cm}^{-1}$ for 12 energy levels. In our work, when gathering all these seven even configurations together in the same model with the fitted parameters taken from Meftah el al. (2017), we found a rather good average energy deviation of $280 \mathrm{~cm}^{-1}$ for the levels of interest, i.e. those involved in the strongest transitions reported in Section 4.

\section{RESULTS AND DISCUSSION}

In view of their potential future use in cosmochronological studies, only the strongest spectral lines in singly ionized uranium were considered in this work. More precisely we limited our investigation to the electric dipole transitions for which the HFR+CPOL log gf values were found to be larger than -1 . It was moreover noticed that most of the weaker transitions, i.e. with $\log g f<-1$, were affected by cancellation effects. As a reminder, in order to calculate the oscillator strength for a transition between the atomic states $\beta J$ and $\beta^{\prime} J^{\prime}$, the line strength has to be computed

$S=\left|\left\langle\beta J|| P^{(1)}|| \beta^{\prime} J^{\prime}\right\rangle\right|^{2}$ 
Table 2. Main $L S$ coupling components of energy levels involved in the transitions listed in Table 1.

\begin{tabular}{|c|c|c|c|c|}
\hline$E\left(\mathrm{~cm}^{-1}\right)^{a}$ & $J$ & $\begin{array}{l}\text { 1st component (per } \\
\text { cent) }\end{array}$ & $\begin{array}{l}\text { 2nd component (per } \\
\text { cent) }\end{array}$ & $\begin{array}{l}\text { 3rd component (per } \\
\text { cent) }\end{array}$ \\
\hline \multicolumn{5}{|l|}{ Odd parity } \\
\hline 0.000 & $9 / 2$ & $77.05 \mathrm{f}^{3} 7 \mathrm{~s}^{2}\left({ }^{4} \mathrm{I}\right)^{4} \mathrm{I}$ & $12.65 \mathrm{f}^{3} 7 \mathrm{~s}^{2}\left({ }^{2} \mathrm{H}\right)^{2} \mathrm{H}$ & $3.65 \mathrm{f}^{3} 6 \mathrm{~d}^{2}\left({ }^{4} \mathrm{I}\right)^{4} \mathrm{I}$ \\
\hline 289.041 & $11 / 2$ & $76.85 \mathrm{f}^{3} 6 \mathrm{~d} 7 \mathrm{~s}\left({ }^{4} \mathrm{I}\right)^{6} \mathrm{~L}$ & $12.95 f^{3} 6 d 7 s\left({ }^{2} H\right)^{4} \mathrm{~K}$ & $4.95 \mathrm{f}^{3} 6 \mathrm{~d} 7 \mathrm{~s}\left({ }^{4} \mathrm{I}\right)^{4} \mathrm{~K}$ \\
\hline 914.765 & $9 / 2$ & $71.35 \mathrm{f}^{3} 6 \mathrm{~d} 7 \mathrm{~s}\left({ }^{4} \mathrm{I}\right)^{6} \mathrm{~K}$ & $10.55 \mathrm{f}^{3} 6 \mathrm{~d} 7 \mathrm{~s}\left({ }^{2} \mathrm{H}\right)^{4} \mathrm{I}$ & $10.25 \mathrm{f}^{3} 6 \mathrm{~d} 7 \mathrm{~s}\left({ }^{4} \mathrm{I}\right)^{4} \mathrm{I}$ \\
\hline 1749.123 & $13 / 2$ & $44.55 \mathrm{f}^{3} 6 \mathrm{~d} 7 \mathrm{~s}\left({ }^{4} \mathrm{I}\right)^{6} \mathrm{~L}$ & $26.15 f^{3} 6 \mathrm{~d} 7 \mathrm{~s}\left({ }^{4} \mathrm{I}\right)^{4} \mathrm{~L}$ & $8.35 \mathrm{f}^{3} 6 \mathrm{~d} 7 \mathrm{~s}\left({ }^{2} \mathrm{H}\right)^{4} \mathrm{~K}$ \\
\hline 2294.696 & $11 / 2$ & $48.35 \mathrm{f}^{3} 6 \mathrm{~d} 7 \mathrm{~s}\left({ }^{4} \mathrm{I}\right)^{6} \mathrm{~K}$ & $17.65 \mathrm{f}^{3} 6 \mathrm{~d} 7 \mathrm{~s}\left({ }^{4} \mathrm{I}\right)^{4} \mathrm{~K}$ & $7.85 \mathrm{f}^{3} 6 \mathrm{~d} 7 \mathrm{~s}\left({ }^{4} \mathrm{I}\right)^{4} \mathrm{I}$ \\
\hline 4585.434 & $13 / 2$ & $28.25 \mathrm{f}^{3} 6 \mathrm{~d}^{2}\left({ }^{4} \mathrm{I}\right)^{6} \mathrm{M}$ & $27.35 f^{3} 6 \mathrm{~d} 7 \mathrm{~s}\left({ }^{4} \mathrm{I}\right)^{6} \mathrm{~L}$ & $14.35 \mathrm{f}^{3} 6 \mathrm{~d} 7 \mathrm{~s}\left({ }^{4} \mathrm{I}\right)^{4} \mathrm{~L}$ \\
\hline 5259.653 & $15 / 2$ & $67.55 \mathrm{f}^{3} 6 \mathrm{~d} 7 \mathrm{~s}\left({ }^{4} \mathrm{I}\right)^{6} \mathrm{~L}$ & $17.85 \mathrm{f}^{3} 6 \mathrm{~d} 7 \mathrm{~s}\left({ }^{4} \mathrm{I}\right)^{4} \mathrm{~L}$ & $6.45 \mathrm{f}^{3} 6 \mathrm{~d} 7 \mathrm{~s}\left({ }^{2} \mathrm{H}\right)^{4} \mathrm{~K}$ \\
\hline 5526.750 & $13 / 2$ & $71.05 \mathrm{f}^{3} 6 \mathrm{~d} 7 \mathrm{~s}\left({ }^{4} \mathrm{I}\right)^{6} \mathrm{~K}$ & $12.35 \mathrm{f}^{3} 6 \mathrm{~d} 7 \mathrm{~s}\left({ }^{4} \mathrm{I}\right)^{4} \mathrm{~K}$ & $5.55 \mathrm{f}^{3} 6 \mathrm{~d} 7 \mathrm{~s}\left({ }^{2} \mathrm{H}\right)^{4} \mathrm{I}$ \\
\hline 6283.431 & $13 / 2$ & $38.45 \mathrm{f}^{3} 6 \mathrm{~d}^{2}\left({ }^{4} \mathrm{I}\right)^{6} \mathrm{M}$ & $21.65 \mathrm{f}^{3} 6 \mathrm{~d} 7 \mathrm{~s}\left({ }^{4} \mathrm{I}\right)^{4} \mathrm{~L}$ & $16.35 \mathrm{f}^{3} 6 \mathrm{~d} 7 \mathrm{~s}\left({ }^{4} \mathrm{I}\right)^{6} \mathrm{~L}$ \\
\hline 8394.362 & $15 / 2$ & $60.45 \mathrm{f}^{3} 6 \mathrm{~d} 7 \mathrm{~s}\left({ }^{4} \mathrm{I}\right)^{6} \mathrm{~K}$ & $14.25 \mathrm{f}^{3} 6 \mathrm{~d}^{2}\left({ }^{4} \mathrm{I}\right)^{6} \mathrm{M}$ & $4.85 \mathrm{f}^{3} 6 \mathrm{~d} 7 \mathrm{~s}\left({ }^{4} \mathrm{I}\right)^{4} \mathrm{~K}$ \\
\hline \multicolumn{5}{|l|}{ Even parity } \\
\hline 22642.478 & $9 / 2$ & $10.45 \mathrm{f}^{3} 7 \mathrm{~s} 7 \mathrm{p}\left({ }^{4} \mathrm{I}\right)^{6} \mathrm{~K}$ & $4.85 \mathrm{f}^{2} 6 \mathrm{~d}^{2} 7 \mathrm{~s}\left({ }^{3} \mathrm{~F}\right)^{4} \mathrm{I}$ & $3.95 \mathrm{f}^{2} 6 \mathrm{~d}^{2} 7 \mathrm{~s}\left({ }^{3} \mathrm{H}\right)^{4} \mathrm{I}$ \\
\hline 23315.092 & $9 / 2$ & $23.75 f^{3} 7 \mathrm{~s} 7 \mathrm{p}\left({ }^{4} \mathrm{I}\right)^{6} \mathrm{~K}$ & $6.05 f^{4} 6 d\left({ }^{5} I\right)^{6} G$ & $5.05 \mathrm{f}^{3} 7 \mathrm{~s} 7 \mathrm{p}\left({ }^{4} \mathrm{I}\right)^{4} \mathrm{I}$ \\
\hline 24288.004 & $11 / 2$ & $7.95 \mathrm{f}^{3} 7 \mathrm{~s} 7 \mathrm{p}\left({ }^{4} \mathrm{I}\right)^{6} \mathrm{~K}$ & $5.55 \mathrm{f}^{3} 7 \mathrm{~s} 7 \mathrm{p}\left({ }^{4} \mathrm{I}\right)^{4} \mathrm{~K}$ & $3.65 f^{4} 6 d\left({ }^{5} I\right)^{6} \mathrm{G}$ \\
\hline 25714.049 & $13 / 2$ & $9.15 \mathrm{f}^{3} 6 \mathrm{~d} 7 \mathrm{p}\left({ }^{4} \mathrm{I}\right)^{6} \mathrm{M}$ & $4.55 \mathrm{f}^{2} 6 \mathrm{~d}^{2} 7 \mathrm{~s}\left({ }^{3} \mathrm{H}\right)^{4} \mathrm{~K}$ & $4.05 \mathrm{f}^{2} 6 \mathrm{~d}^{2} 7 \mathrm{~s}\left({ }^{3} \mathrm{H}\right)^{4} \mathrm{~L}$ \\
\hline 25967.697 & $7 / 2$ & $19.75 f^{3} 7 \mathrm{~s} 7 \mathrm{p}\left({ }^{4} I\right)^{6} \mathrm{I}$ & $5.35 f^{2} 6 d 7 s^{2}\left({ }^{3} F\right)^{4} H$ & $3.75 \mathrm{f}^{3} 7 \mathrm{~s} 7 \mathrm{p}\left({ }^{4} \mathrm{I}\right)^{4} \mathrm{H}$ \\
\hline 26191.312 & $13 / 2$ & $21.75 f^{3} 6 \mathrm{~d} 7 \mathrm{p}\left({ }^{4} \mathrm{I}\right)^{6} \mathrm{M}$ & $8.95 \mathrm{f}^{2} 6 \mathrm{~d}^{2} 7 \mathrm{~s}\left({ }^{3} \mathrm{H}\right)^{4} \mathrm{~K}$ & $8.05 \mathrm{f}^{3} 6 \mathrm{~d} 7 \mathrm{p}\left({ }^{4} \mathrm{I}\right)^{4} \mathrm{~L}$ \\
\hline 26415.115 & $13 / 2$ & $8.15 \mathrm{f}^{2} 6 \mathrm{~d}^{2} 7 \mathrm{~s}\left({ }^{3} \mathrm{H}\right)^{6} \mathrm{H}$ & $7.15 f^{3} 6 d 7 p\left({ }^{4} I\right)^{6} M$ & $5.55 \mathrm{f}^{2} 6 \mathrm{~d}^{2} 7 \mathrm{~s}\left({ }^{3} \mathrm{H}\right)^{4} \mathrm{~K}$ \\
\hline 26716.697 & $13 / 2$ & $7.25 f^{4} 6 d\left({ }^{5} F\right)^{6} \mathrm{H}$ & $5.65 \mathrm{f}^{2} 6 \mathrm{~d}^{2} 7 \mathrm{~s}\left({ }^{3} \mathrm{H}\right)^{4} \mathrm{~K}$ & $5.35 \mathrm{f}^{2} 6 \mathrm{~d}^{2} 7 \mathrm{~s}\left({ }^{1} \mathrm{G}\right)^{4} \mathrm{~K}$ \\
\hline 27929.924 & $11 / 2$ & $5.65 \mathrm{f}^{2} 6 \mathrm{~d} 7 \mathrm{~s}^{2}\left({ }^{3} \mathrm{H}\right)^{4} \mathrm{H}$ & $5.35 \mathrm{f}^{3} 7 \mathrm{~s} 7 \mathrm{p}\left({ }^{4} \mathrm{I}\right)^{6} \mathrm{~K}$ & $4.55 \mathrm{f}^{3} 6 \mathrm{~d} 7 \mathrm{p}\left({ }^{4} \mathrm{I}\right)^{6} \mathrm{~L}$ \\
\hline 28154.447 & $11 / 2$ & $7.05 \mathrm{f}^{3} 6 \mathrm{~d} 7 \mathrm{p}\left({ }^{4} \mathrm{I}\right)^{6} \mathrm{~L}$ & $6.55 \mathrm{f}^{4} 7 \mathrm{~s}\left({ }^{3} \mathrm{H}\right)^{4} \mathrm{H}$ & $3.55 \mathrm{f}^{4} 7 \mathrm{~s}\left({ }^{3} \mathrm{H}\right)^{2} \mathrm{H}$ \\
\hline 28322.361 & $11 / 2$ & $8.15 f^{3} 6 \mathrm{~d} 7 \mathrm{p}\left({ }^{4} \mathrm{I}\right)^{6} \mathrm{~L}$ & $4.75 \mathrm{f}^{2} 6 \mathrm{~d}^{2} 7 \mathrm{~s}\left({ }^{3} \mathrm{~F}\right)^{6} \mathrm{H}$ & $3.65 \mathrm{f}^{2} 6 \mathrm{~d}^{2} 7 \mathrm{~s}\left({ }^{1} \mathrm{G}\right)^{4} \mathrm{~K}$ \\
\hline 28507.894 & $11 / 2$ & $5.05 f^{4} 6 d\left({ }^{5} G\right)^{6} I$ & $4.05 \mathrm{f}^{4} 7 \mathrm{~s}\left({ }^{3} \mathrm{H}\right)^{4} \mathrm{H}$ & $3.75 \mathrm{f}^{2} 6 \mathrm{~d} 7 \mathrm{~s}^{2}\left({ }^{3} \mathrm{H}\right)^{4} \mathrm{H}$ \\
\hline 28587.261 & $11 / 2$ & $7.35 f^{3} 6 \mathrm{~d} 7 \mathrm{p}\left({ }^{4} \mathrm{I}\right)^{6} \mathrm{~L}$ & $5.35 \mathrm{f}^{2} 6 \mathrm{~d}^{2} 7 \mathrm{~s}\left({ }^{3} \mathrm{~F}\right)^{6} \mathrm{H}$ & $3.65 \mathrm{f}^{4} 7 \mathrm{~s}\left({ }^{1} \mathrm{H}\right)^{2} \mathrm{H}$ \\
\hline 29206.703 & $11 / 2$ & $6.65 \mathrm{f}^{2} 6 \mathrm{~d}^{3}\left({ }^{3} \mathrm{H}\right){ }^{6} \mathrm{~K}$ & $3.15 \mathrm{f}^{2} 6 \mathrm{~d}^{3}\left({ }^{3} \mathrm{H}\right)^{4} \mathrm{I}$ & $2.65 \mathrm{f}^{2} 6 \mathrm{~d}^{2} 7 \mathrm{~s}\left({ }^{3} \mathrm{~F}\right)^{6} \mathrm{G}$ \\
\hline 29476.743 & $13 / 2$ & $12.45 f^{3} 7 \mathrm{~s} 7 \mathrm{p}\left({ }^{4} \mathrm{I}\right)^{6} \mathrm{~K}$ & $5.15 \mathrm{f}^{2} 6 \mathrm{~d}^{2} 7 \mathrm{~s}\left({ }^{3} \mathrm{H}\right)^{6} \mathrm{I}$ & $2.95 \mathrm{f}^{3} 7 \mathrm{~s} 7 \mathrm{p}\left({ }^{4} \mathrm{I}\right)^{4} \mathrm{~K}$ \\
\hline 29932.395 & $15 / 2$ & $9.75 \mathrm{f}^{2} 6 \mathrm{~d} 7 \mathrm{~s}^{2}\left({ }^{3} \mathrm{H}\right)^{4} \mathrm{I}$ & $5.15 f^{4} 6 d\left({ }^{5} F\right)^{6} \mathrm{H}$ & $5.05 \mathrm{f}^{3} 6 \mathrm{~d} 7 \mathrm{p}\left({ }^{4} \mathrm{I}\right)^{6} \mathrm{M}$ \\
\hline 29936.466 & $11 / 2$ & $11.95 f^{3} 6 \mathrm{~d} 7 \mathrm{p}\left({ }^{4} \mathrm{I}\right)^{6} \mathrm{~L}$ & $7.65 f^{2} 6 d^{2} 7 s\left({ }^{3} F\right)^{6} G$ & $4.75 \mathrm{f}^{2} 6 \mathrm{~d}^{2} 7 \mathrm{~s}\left({ }^{3} \mathrm{H}\right)^{4} \mathrm{G}$ \\
\hline 29978.143 & $13 / 2$ & $13.95 \mathrm{f}^{2} 6 \mathrm{~d}^{3}\left({ }^{3} \mathrm{H}\right)^{6} \mathrm{~K}$ & $5.15 \mathrm{f}^{2} 6 \mathrm{~d}^{2} 7 \mathrm{~s}\left({ }^{3} \mathrm{H}\right)^{6} \mathrm{I}$ & $3.75 \mathrm{f}^{3} 7 \mathrm{~s} 7 \mathrm{p}\left({ }^{4} \mathrm{I}\right)^{6} \mathrm{~K}$ \\
\hline 30060.727 & $11 / 2$ & $5.05 \mathrm{f}^{3} 6 \mathrm{~d} 7 \mathrm{p}\left({ }^{4} \mathrm{I}\right)^{6} \mathrm{~L}$ & $4.45 \mathrm{f}^{2} 6 \mathrm{~d}^{2} 7 \mathrm{~s}\left({ }^{3} \mathrm{H}\right)^{6} \mathrm{G}$ & $2.75 f^{4} 6 d\left({ }^{5} F\right)^{4} G$ \\
\hline 30240.416 & $11 / 2$ & $3.05 \mathrm{f}^{4} 7 \mathrm{~s}\left({ }^{3} \mathrm{H}\right)^{2} \mathrm{H}$ & $2.85 f^{3} 6 \mathrm{~d} 7 \mathrm{p}\left({ }^{4} \mathrm{I}\right)^{6} \mathrm{~L}$ & $2.25 \mathrm{f}^{2} 6 \mathrm{~d} 7 \mathrm{~s}^{2}\left({ }^{3} \mathrm{H}\right)^{4} \mathrm{H}$ \\
\hline 30263.978 & $9 / 2$ & $11.65 f^{3} 7 \mathrm{~s} 7 \mathrm{p}\left({ }^{4} I\right)^{6} \mathrm{I}$ & $3.45 \mathrm{f}^{2} 6 \mathrm{~d}^{3}\left({ }^{3} \mathrm{H}\right)^{6} \mathrm{I}$ & $3.45 f^{3} 6 \mathrm{~d} 7 \mathrm{p}\left({ }^{4} \mathrm{I}\right)^{6} \mathrm{~K}$ \\
\hline 30341.673 & $15 / 2$ & $18.45 f^{3} 6 \mathrm{~d} 7 \mathrm{p}\left({ }^{4} \mathrm{I}\right)^{6} \mathrm{M}$ & $12.45 \mathrm{f}^{4} 7 \mathrm{~s}\left({ }^{1} \mathrm{~K}\right)^{2} \mathrm{~K}$ & $5.25 \mathrm{f}^{2} 6 \mathrm{~d}^{2} 7 \mathrm{~s}\left({ }^{3} \mathrm{H}\right)^{2} \mathrm{~L}$ \\
\hline
\end{tabular}

Note. ${ }^{a}$ Experimental energy levels from Blaise et al. (1994) and Meftah et al. (2017).

or its square root

$S^{1 / 2}=\left\langle\beta J\left\|P^{(1)}\right\| \beta^{\prime} J^{\prime}\right\rangle$,

where $P^{(1)}$ corresponds to the electric dipole operator. The wavefunctions are expanded in terms of basis functions due to intermediate coupling and configuration interaction mixing:

$\left|\beta J>=\sum_{\gamma} y_{\gamma J}^{\beta}\right| \gamma J>$

$\left|\beta^{\prime} J^{\prime}>=\sum_{\gamma^{\prime}} y_{\gamma^{\prime} J^{\prime}}^{\beta^{\prime}}\right| \gamma^{\prime} J^{\prime}>$

We may then write (7) in the form

$S^{1 / 2}=\sum_{\gamma} \sum_{\gamma^{\prime}} y_{\gamma J}^{\beta}\left\langle\gamma J\left\|P^{(1)}\right\| \gamma^{\prime} J^{\prime}\right\rangle y_{\gamma^{\prime} J^{\prime}}^{\beta^{\prime}}$

This sum represents a mixing of amplitudes that frequently leads to destructive interference effects, in particular for weak lines. In making numerical calculations from (10), it is thus worthwhile evaluating a cancellation factor

$\mathrm{CF}=\left[\frac{\left|\sum_{\gamma} \sum_{\gamma^{\prime}} y_{\gamma J}^{\beta}<\gamma J \| P^{(1)}\right|\left|\gamma^{\prime} J^{\prime}>y_{\gamma^{\prime} J^{\prime}}^{\beta^{\prime}}\right|}{\sum_{\gamma} \sum_{\gamma^{\prime}}\left|y_{\gamma J}^{\beta}<\gamma J \| P^{(1)}\right|\left|\gamma^{\prime} J^{\prime}>y_{\gamma^{\prime} J^{\prime}}^{\beta^{\prime}}\right|}\right]^{2}$.
According to Cowan (1981), very small CF values (typically smaller than about 0.05 ) indicate that the corresponding line strengths may be expected to show large uncertainties. In Fig. 3, cancellation factors are plotted as a function of $\log g f$ for all $\mathrm{U}_{\mathrm{II}}$ transitions. We can clearly note that most of lines with $\log g f<$ -1 are affected by very small $\mathrm{CF}$ values indicating that the corresponding oscillator strengths could be unreliable. On the contrary, stronger transitions, with $\log g f>-1$, do not appear to be affected by cancellation effects.

Such strong transitions, involving energy levels up to $30342 \mathrm{~cm}^{-1}$, are reported in Table 1 . This corresponds to $38 \mathrm{U}$ II spectral lines appearing in the wavelength range from 3337 and $4627 \AA$. For these lines, our $\log g f$ values are compared with the 'old' data deduced from emission line intensity measurements by Corliss (1976), Chen and Borzileri (1981), Henrion et al. (1987) and Kurucz (1995), as well as with the more recent and more accurate experimental values obtained by Nilsson et al. (2002). It can be observed, from Table 1, that our calculated oscillator strengths are in reasonable agreement with the latter measurements, the mean relative difference between both sets of $g f$ values being found to be equal to 25 per cent if we except four lines at 3670.068, 3865.916, 4155.409, and $4241.664 \AA$, for which our HFR+CPOL results are about a factor of 2 smaller than the experimental oscillator strengths of Nilsson et a (2002). It is worth noting however that, three of these 


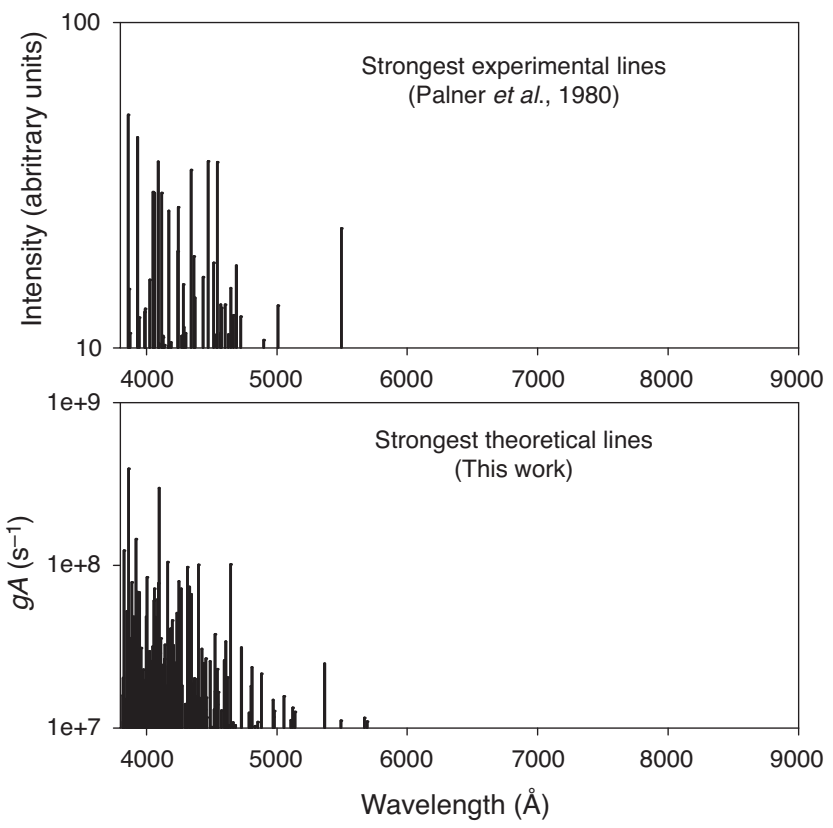

Figure 4. Comparison between experimental and theoretical strongest emission lines in U II. Only visible spectral lines observed by Palmer et al. (1980) with measured intensities greater than 10 (top figure) and those calculated in this work with weighted transition probabilities, $g A$, larger than $10^{7} \mathrm{~s}^{-1}$ (bottom figure) are shown.

transitions are characterized by the same upper even-parity level at $28154.451 \mathrm{~cm}^{-1}$, which was found to be extremely mixed, and thus very sensitive to small changes in the eigenvector composition. Indeed, for this level, our calculations led to a main $L S$ component, i.e. $5 f^{3}\left({ }^{4} I\right) 6 d 7 p{ }^{6} L_{11 / 2}$, as weak as 7 per cent, to be compared with 18 per cent obtained by Meftah et al. (2017) using a much more limited physical model.

For four other spectral lines, at $\lambda=3337.785,3623.057$, 4172.973, and $4178.995 \AA$, our computed $\log g f$ values are given between square brackets in Table 1 to indicate that the corresponding results are likely to be affected by larger uncertainties. This is due to the fact that, for the two upper even-parity levels involved in these transitions, it was extremely difficult to establish a trustworthy correspondence between the experimental values, i.e. $E=28507.894$ and $30240.416 \mathrm{~cm}^{-1}$, and the calculated ones, the retained theoretical levels being moreover very strongly mixed, with main $L S$ components not exceeding 5 per cent, according to our calculations. These two levels were by the way not classified in the recent parametric analysis of the $U_{\text {II }}$ spectrum by Meftah et al. (2017).

The strength of intermediate coupling and configuration interaction characterizing the $\mathrm{U}$ II energy level structure is illustrated in Table 2 where the first three $L S$ components, as calculated in this work, are given for all the levels involved in the transitions listed in Table 1. It is clear that the majority of levels appear to be extremely mixed, preventing any reliable spectroscopic designation. This is particularly the case for the even parity in which many levels were found to be the mixture of numerous significant contributions (sometimes up to 20) of comparable amplitudes.

A qualitative comparison between the strongest experimental and theoretical transitions is shown in Fig. 4, in which all the $U_{\text {II }}$ visible spectral lines observed by Palmer et al. (1980) with measured intensities greater than 10 are compared to those calculated in this work with weighted transition probabilities, $g A$, larger than $10^{7} \mathrm{~s}^{-1}$. It is clearly seen that the overall agreement between both spectra is very satisfactory.

It is also particularly interesting to consider the line at 3859.571 $\AA$, which was used as cosmochronometer by Cayrel et al. (2001a). For this line, our $g f$ value of 0.875 is in excellent agreement with the accurate experimental result reported by Nilsson et al. (2002), i.e. $g f=0.857$, whereas all the previous data were very scattered between $g f$ values so different as 0.240 (Corliss 1976), 0.625 (Chen and Borzileri 1981), 0.190 (Henrion et al. 1987), and 0.785 (Kurucz 1995).

Finally, it was found that CPOL effects considered in our physical model have a major influence on the final oscillator strengths, the mean ratio $g f_{\mathrm{HFR}}+\mathrm{CPOL} / g f_{\mathrm{HFR}}$ being found to be equal to 0.57 . These effects, which are assumed to take account of the most important core-valence and core-core correlations, are expected to be much larger than those of intravalence correlations not explicitly considered in our multiconfiguration expansions. It was indeed verified that our computed transition rates were slightly sensitive to additional valence configurations. More precisely, in a first step, the configurations $5 \mathrm{f}^{2} 7 \mathrm{~s}^{2} 7 \mathrm{p}, 5 \mathrm{f}^{2} 6 \mathrm{~d} 7 \mathrm{~s} 7 \mathrm{p}, 5 \mathrm{f}^{2} 6 \mathrm{~d}^{2} 7 \mathrm{p}, 5 \mathrm{f}^{2} 7 \mathrm{~s} 7 \mathrm{p}^{2}$, and $5 \mathrm{f}^{2} 6 \mathrm{~d} 7 \mathrm{p}^{2}$ were investigated. By comparing a nine-configuration ab intio HFR calculation (including $5 \mathrm{f}^{3} 7 \mathrm{~s}^{2}, 5 \mathrm{f}^{3} 6 \mathrm{~d} 7 \mathrm{~s}, 5 \mathrm{f}^{3} 6 \mathrm{~d}^{2}, 5 \mathrm{f}^{4} 7 \mathrm{~s}$, $5 f^{4} 6 d, 5 f^{2} 6 d 7 s^{2}, 5 f^{2} 6 d^{2} 7 s, 5 f^{3} 7 s 7 p$, and $\left.5 f^{3} 6 d 7 p\right)$ with a fourteenconfiguration calculation (adding $5 \mathrm{f}^{2} 7 \mathrm{~s}^{2} 7 \mathrm{p}, 5 \mathrm{f}^{2} 6 \mathrm{~d} 7 \mathrm{~s} 7 \mathrm{p}, 5 \mathrm{f}^{2} 6 \mathrm{~d}^{2} 7 \mathrm{p}$, $5 f^{2} 7 \mathrm{~s} 7 \mathrm{p}^{2}$, and $5 \mathrm{f}^{2} 6 \mathrm{~d} 7 \mathrm{p}^{2}$ ) the effect of the latter five configurations was estimated and found rather small. Indeed, the mean difference for the strongest lines $(\log g f>-1)$ was 0.09 dex, which corresponds to a relative discrepancy of a few percent. When adding $5 f^{3} 7 \mathrm{~s} 7 \mathrm{~d}, 5 \mathrm{f}^{3} 6 \mathrm{~d} 7 \mathrm{~d}, 5 \mathrm{f}^{4} 7 \mathrm{~d}, 5 \mathrm{f}^{2} 7 \mathrm{~s}^{2} 7 \mathrm{~d}$, and $5 \mathrm{f}^{2} 6 \mathrm{~d}^{2} 7 \mathrm{~d}$ to the nine-configuration expansion, the mean difference was then 0.05 dex. Finally, the inclusion of the $5 f^{3} 7 \mathrm{~s} 8 \mathrm{~s}, 5 \mathrm{f}^{3} 6 \mathrm{~d} 8 \mathrm{~s}, 5 \mathrm{f}^{4} 8 \mathrm{~s}, 5 \mathrm{f}^{2} 6 \mathrm{~d}^{2} 8 \mathrm{~s}$, $5 \mathrm{f}^{2} 7 \mathrm{~s}^{2} 8 \mathrm{~s}, 5 \mathrm{f}^{3} 7 \mathrm{~s} 8 \mathrm{p}$, and $5 \mathrm{f}^{3} 6 \mathrm{~d} 8 \mathrm{p}$ was found to affect the $\log g f$ values by less than 0.04 dex.

\section{CONCLUSION}

This work represents the first attempt to establish a reliable list of $U$ II spectral lines that could be used in cosmochronology, corresponding to 38 strong electric dipole transitions in the wavelength visible region. To do so, a pseudo-relativistic HFR model including CPOL corrections was employed to compute the oscillator strengths, the latter being found in satisfactory overall agreement with the most accurate experimental data, where available. In view of the complexity of the U II spectrum, it is clear, however, that additional efforts, on both the experimental and theoretical sides, are still needed for improving the accuracy of the radiative parameters in this spectrum of high cosmochronological interest.

\section{ACKNOWLEDGEMENTS}

PP and PQ are, respectively, Research Associate and Research Director of the Belgian Fund for Scientific Research FRS-FNRS. Financial support from this organization is gratefully acknowledged.

\section{REFERENCES}

Biémont E. et al., 2001, MNRAS, 321, 481

Biémont E., Quinet P., Ryabchikova T. A., 2002, MNRAS, 336, 1155 
Blaise J., Wyart J.-F., 1992, Selected Constants Energy Levels and Atomic Spectra of Actinides Vol. 22. Centre National de la Recherche Scientifique, Paris

Blaise J., Wyart J.-F., Vergés J., Engleman R., Jr, Palmer B. A., Radziemski L. J., 1994, J. Opt. Soc. Am. B, 11, 1897

Brewer L., 1971, J. Opt. Soc. Am., 12, 1666

Butcher H. R., 1987, Nature, 328, 127

Cayrel R. et al., 2001a, Nature, 409, 691

Cayrel R. et al., 2001b, in von Hippel T., Simpson C., Manset N., eds, ASP Conf. Ser., Vol. 245: Astrophysical Ages and Times Scales. Astron. Soc. Pac., San Francisco, p. 244

Chen H. L., Borzileri C., 1981, J. Chem. Phys., 74, 6063

Corliss C. H., 1976, J. Res. Natl. Bur. Stand., 80A, 429

Corliss C. H., Bozman W. R., 1962, NBS Monograph 53. US Government Printing Office, Washington DC

Cowan R. D., 1981, The Theory of Atomic Structure and Spectra. California Univ. Press, Berkeley

Cowan J. J., Pfeiffer B., Kratz K. L., Thielemann F. K., Sneden C., Burles S., Tytler D., Beers T. C., 1999, ApJ, 521, 194

Fraga S., Karwowski J., Saxena K. M. S., 1976, Handbook of Atomic Data. Elsevier, Amsterdam

François P., Spite M., Spite F., 1993, A\&A, 274, 821

Goriely S., Clerbaux B., 1999, A\&A, 346, 798

Hameed S., 1972, J. Phys. B, 5, 746

Hameed S., Herzenberg A., James M. G., 1968, J. Phys. B, 1, 822

Henrion G., Fabry M., Remy M., 1987, J. Quant. Spectrosc. Radiat. Transfer., 37,477
Hibbert A., 1982, Nucl. Instrum. Methods Phys. Res., 202, 323

Kurucz R. L., 1995, Atomic Spectral line Database from CD-ROM 23. Available online at https://www.cfa.harvard.edu/amp/ampdata/kurucz23/sek ur.html (accessed in 2018 April)

Li Z. S. et al., 2001, J. Phys. B, 34, 1349

Lundberg H., Johansson S., Nilsson H., Zhang Z., 2001, A\&A, 372, L50

Meftah A., Sabri M., Wyart J. F., Tchang-Brillet W. Ü., 2017, Atoms, 5, 24

Meggers W. F., Corliss C. H., Schribner B. F., 1961, NBS Monograph 32. US Government Printing Office, Washington DC

Nilsson H., Ivarsson S., Johansson S., Lundberg H., 2002, A\&A, 381, 1090 Palmer B. A., Keller R. A., Engleman R.,, Jr , 1980, LASL Informal Report LA-8251-MS, UC-34a. Los Alamos Scientific Laboratory, NM, USA

Quinet P., 2017, Can. J. Phys., 95, 790

Quinet P., Palmeri P., Biémont E., McCurdy M. M., Rieger G., Pinnington E. H., Wickliffe M. E., Lawler J. E., 1999, MNRAS, 307, 934

Quinet P., Palmeri P., Biémont E., Li Z. S., Zhang Z. G., Svanberg S., 2002, J. Alloys Compd., 344, 255

Steinhaus D. W., Radziemski L. J.,, Jr, Cowan R. D., Blaise J., Guelachvili G., Ben Osman Z., Vergès J., 1971, LASL Report LA-4501, UC-34. Los Alamos Scientific Laboratory, NM, USA

Voigt P. A., 1975, Phys. Rev. A, 11, 1845

Zhang Z. G., Li Z. S., Svanberg S., Palmeri P., Quinet P., Biémont E., 2001, Eur. Phys. J. D, 15, 301

This paper has been typeset from a $\mathrm{T}_{\mathrm{E}} \mathrm{X} / \mathrm{L} \mathrm{T} \mathrm{T}_{\mathrm{E}} \mathrm{X}$ file prepared by the author. 